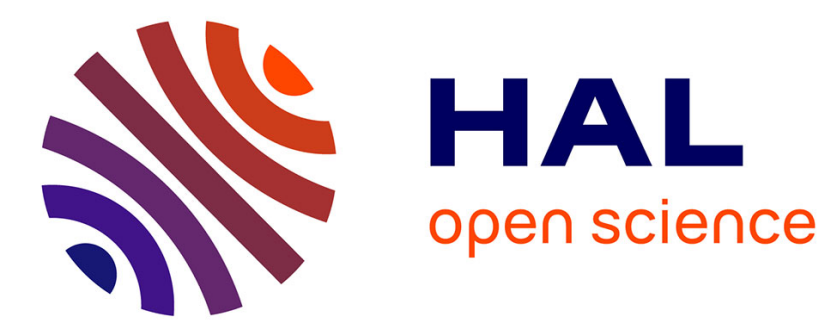

\title{
Phase Behavior of Nanoparticles in a Thermotropic Liquid Crystal
}

Cristina da Cruz, Olivier Sandre, Valérie Cabuil

\section{To cite this version:}

Cristina da Cruz, Olivier Sandre, Valérie Cabuil. Phase Behavior of Nanoparticles in a Thermotropic Liquid Crystal. Journal of Physical Chemistry B, 2005, 109 (30), pp.14292-14299. 10.1021/jp0455024 . hal-00016296

\section{HAL Id: hal-00016296 https://hal.science/hal-00016296}

Submitted on 24 Oct 2018

HAL is a multi-disciplinary open access archive for the deposit and dissemination of scientific research documents, whether they are published or not. The documents may come from teaching and research institutions in France or abroad, or from public or private research centers.
L'archive ouverte pluridisciplinaire HAL, est destinée au dépôt et à la diffusion de documents scientifiques de niveau recherche, publiés ou non, émanant des établissements d'enseignement et de recherche français ou étrangers, des laboratoires publics ou privés. 


\title{
Phase behavior of nanoparticles in a thermotropic liquid crystal
}

\author{
Cristina Da Cruz, Olivier Sandre, Valérie Cabuil* \\ Laboratoire des Liquides Ioniques et Interfaces Chargées, Unité Mixte de Recherche 7612 Centre National de la Recherche \\ Scientifique / Université Pierre et Marie Curie,
}

Case 63, 4 Place Jussieu, 75252 Paris cedex 05 FRANCE

* corresponding author

Received: October 4, 2004; In Final Form: May 24, 2005

Keywords : ferronematics, ferrofluids, nanoparticles, liquid crystals, SAXS

ABSTRACT: In this work we describe the outstanding behavior of a nanocomposite system composed of the thermotropic liquid crystal 5CB doped with nanoparticles of the magnetic iron oxide maghemite $\left(\gamma-\mathrm{Fe}_{2} \mathrm{O}_{3}\right)$. We show that the I-N transition is associated to a reversible gathering of nanoparticles inside droplets of ferronematic phase coexisting with a non magnetic nematic host phase.

Composite systems composed of a liquid crystal (LC) doped by particles have been the subject of numerous famous works in the past ${ }^{1}$ and in the last years. ${ }^{2}$ LC ordering induces topological defects ${ }^{3,4}$ and additional long-range forces ${ }^{5}$ among colloidal particles. These forces can result in the formation of supermolecular structures, ${ }^{6,7}$ cellular structures ${ }^{8,9}$ and even soft solids. ${ }^{10}$

Due to their anisotropic properties, liquid crystals can be orientated under magnetic or electric fields. ${ }^{11}$ However, the intensity of a magnetic field necessary to align a LC can be too high (> 0.1 Tesla) for practical applications. This limitation could be overcome with ferronematic liquid crystals (FNs) obtained by doping a nematic matrix with ferromagnetic particles. Well before their real experimental synthesis, ferronematics were first introduced theoretically by Brochard and de Gennes ${ }^{1}$ to increase the magnetic anisotropy of LCs and to lower the magnetic field intensities required for their orientation. The authors also predicted that a strong anchoring of the mesogen molecules onto the particles would result in a ferromagnetic behavior of the matrix (i.e. a hysteretic magnetization curve). ${ }^{1}$

Since then, research on lyotropic ${ }^{12-15}$ or thermotropic ${ }^{16,17}$ ferronematics have focused on the decrease of the critical field of the so-called Fredericks transition, which originates from the competition between field-induced and surfaceinduced orientations of the nematic director. Until now, the poor solubility of the magnetic grains in thermotropic LCs has restricted their study to either large $(0.5 \mu \mathrm{m}$ long $)$ magnetic needles ${ }^{16}$ or to spherical magnetic nanoparticles (with typical dimensions of $100 \AA$ ) but volume fractions of $0.05 \%$ at most $^{17}$.

Ferrofluids are colloidal suspensions of magnetic nanoparticles that remain stable under whatever intensity of magnetic field or magnetic field gradient. ${ }^{18}$ So-called ionic ferrofluids ${ }^{19,20}$ are composed of electrically charged magnetic grains in water, which electrostatic repulsion prevents from flocculation. A second type called oily ferrofluids is composed of surfactant coated magnetic nanoparticles, for which the stability of the colloidal solution is provided by a steric repulsion which counterbalances Van der Waals and magnetic dipolar attractions. Ferrofluids in various organic solvents can be derived from ionic ferrofluids by coating the grains with appropriate surfactants. ${ }^{21}$ The typical concentration of magnetic grains in a ferrofluid is larger than $10^{16}$ grains $/ \mathrm{cm}^{3}$. The average magnetic moment of a grain is of the order of $10^{4}$ Bohr magnetons (also the approximate number of iron ions per nanoparticle).

Herein we describe the formulation of a composite material that results from the incorporation of magnetic nanoparticles into a matrix made of a thermotropic liquid crystal (LC) exhibiting a nematic phase at room temperature. A special attention is paid to the dispersion state and to the phase behavior of the magnetic nanoparticles in the matrix in relation with the phase behavior of the LC.

Previous works mentioned dispersing a magnetic powder made of nanometric grains directly into a liquid crystal and then sonicating ${ }^{17}$. Instead of this method, we chose to mix the LC with a ferrofluid constituted of a dispersion of magnetic nanoparticles in a volatile solvent in order to reach higher concentrations in the LC and a proper dispersion state. The choice of the surfactant preventing particles from aggregation is of uppermost importance.

\section{Materials}

The nematic liquid crystal used for this work is $5 \mathrm{CB}$ i. e. 4 pentyl-4'-cyano-biphenyl presented in scheme 1 , which exhibits a nematic phase between $21^{\circ} \mathrm{C}$ and $35^{\circ} \mathrm{C}$.

The magnetic nanoparticles are prepared by alkaline coprecipitation of ferric and ferrous salts following a procedure described previously. ${ }^{20}$ The obtained magnetic oxide is maghemite $\gamma-\mathrm{Fe}_{2} \mathrm{O}_{3}$. The surface of the nanoparticles is grafted by Beycostat NE (BNE), ${ }^{21,22}$ which is a commercial anionic surfactant composed of a mixture of mono- and di-esters of phosphoric acid, the chain being an alkylphenol containing nine ethoxy groups. We choose Beycostat NE to coat the nanoparticles because of its affinity for aromatic solvents, whereas oleic acid used in some publications leads to a systematic aggregation of the magnetic nanoparticles. BNE is 
This document is the Accepted Manuscript version of a Published Work that appeared in final form in see J. Phys. Chem. B 2005, 109, 14292-14299, copyright (C) American Chemical Society after peer review and technical editing by the publisher. To access the final edited and published work see http://pubs.acs.org/doi/abs/10.1021/jp0455024

adsorbed directly onto the precursor cationic maghemite nanoparticles in nitric acid medium. After rinsing some of the ungrafted BNE in excess in a mixture of methanol/ether, the dispersion of the nanoparticles is achieved in dichloromethane. These magnetic nanoparticles exhibit some polydispersity in diameters ranging from 5 to $12 \mathrm{~nm}$. (see supporting information)

Scheme 1: Molecular structure and polymorphism of 5CB, the liquid crystal here in study.

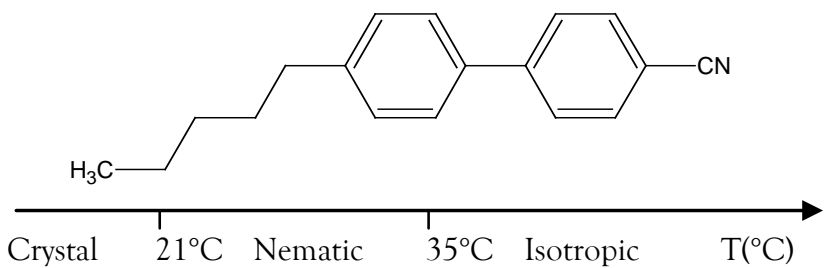

Preparation of ferronematic samples

The ferronematics are prepared by simply adding the magnetic nanoparticles suspended in dichloromethane to the liquid crystal in nematic phase at room temperature and then waiting for the solvent to evaporate. The iron concentration in the LC is measured by Atomic Absorption Spectroscopy (AAS) at $\lambda=480 \mathrm{~nm}$ after degradation of iron oxide nanoparticles into ferric ions by concentrated hydrochloric acid. ${ }^{23}$

\section{Optical observations}

First we see by eye a biphasic state at room temperature, that disappears when we heat carefully our ferronematics until crossing the phase transition to isotropic state. At this $\mathrm{T}_{(\mathrm{N}-1)}$ temperature and above, a stable dispersion of the nanoparticles is obtained. This homogeneous mixture resulting from the heating to the isotropic phase stays homogeneous even when submitted to a strong magnetic field (around 1 Tesla) as long as temperature remains above $\mathrm{T}_{(\mathrm{N}-1)}$. Therefore, it can be considered as a true ferrofluid with the isotropic LC as solvent. Optical microscopy experiments are carried out in order to observe the microphase behavior of the mixture.

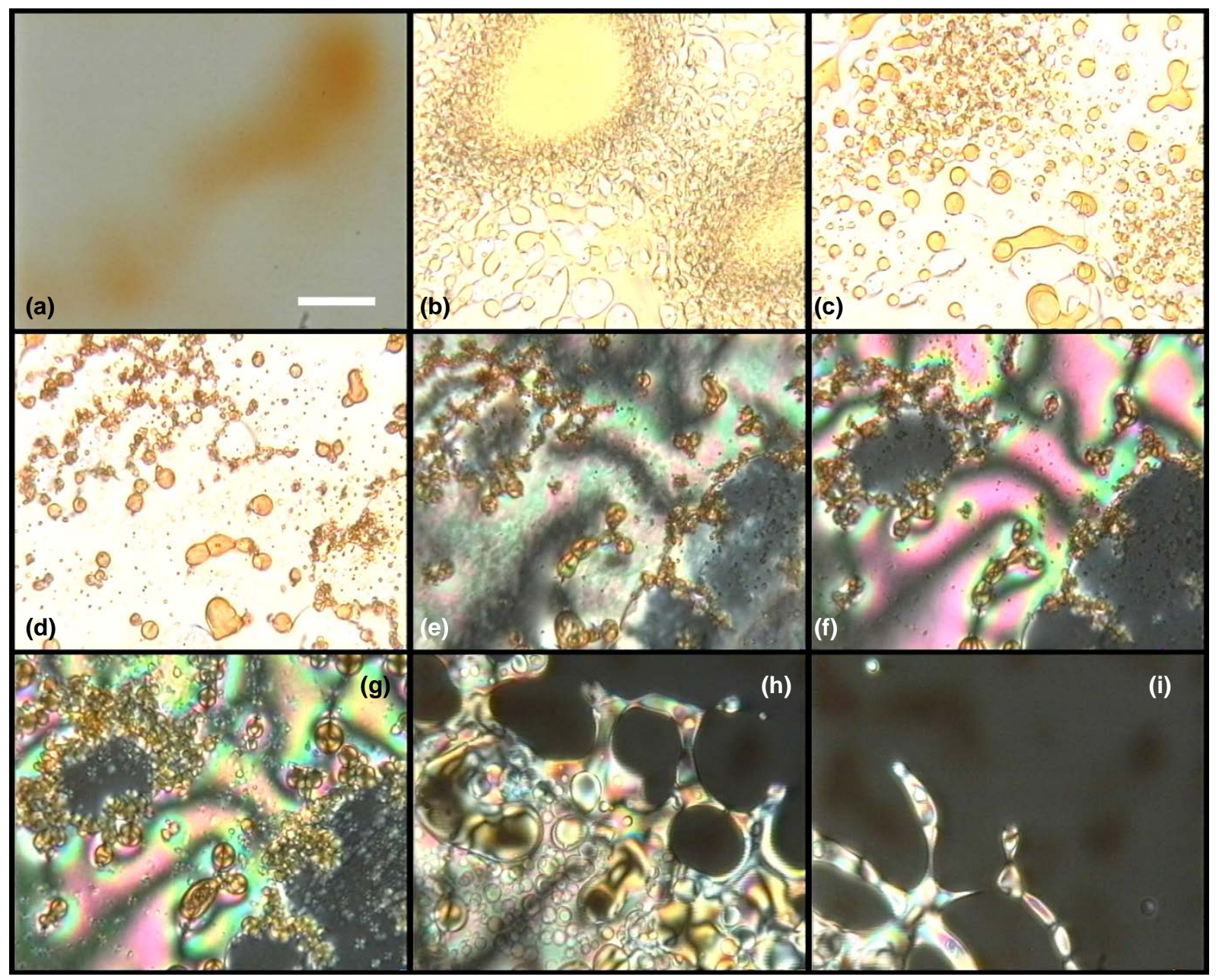

Figure 1: Observation of the microphase separation of a typical ferronematic sample of this study by optical microscopy under parallel

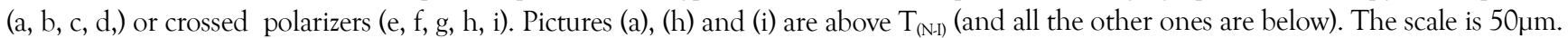
(a): isotropic phase above T(N-I) in an almost completely homogeneous state, the orange color being slowly disappearing; (b): sudden apparition of an interface at T(N-I); (c): formation of round droplets; (d): shrinking of the droplets and concentrating of the magnetic materials inside; (e): observation of the strings of droplets superposed with the disinclination lines of the nematic matrix; (f): observation of black crosses inside the droplets; (g): growth of the droplets when the temperature is increased; (h): above T(N-I), dissolution of the droplets into the nematic and advance of the isotropic (black) phase; (i): homogeneous isotropic state almost everywhere.

The FN samples are observed under crossed polarizers using an upright microscope (Leitz, 20X magnification) equipped with a homemade Peltier temperature-controlled stage. Some of the warm isotropic mixture is deposited between two glass slides where it cools down and then it is heated again above the nematic-isotropic transition point. The phenomenon 
This document is the Accepted Manuscript version of a Published Work that appeared in final form in see J. Phys. Chem. B 2005, 109, 14292-14299, copyright (C) American Chemical Society after peer review and technical editing by the publisher. To access the final edited and published work see http://pubs.acs.org/doi/abs/10.1021/jp0455024

occurring on cooling below the I-N transition appears under the microscope as the formation of dark orange microdroplets, which means rich in iron oxide nanoparticles. In a reversible way, droplets disappear abruptly on heating above $\mathrm{T}_{(\mathrm{N}-1)}$, and then are replaced by a diffuse cloud of nanoparticles vanishing slowly (figure 1a). On decreasing again temperature below the I-N transition, the microphase separation again occurs in a reversible way and at the same temperature. After a fluctuating interface has appeared suddenly from the initially homogenous matrix (figure 1b), the new phase concentrates into droplets, that rapidly shrink and turn into a darker color (figure 1c). We observe also that the neighboring droplets originating from the rupture of a larger drop remain aligned together (figure 1d). Under crossed polarizers (figure 1e), the localization of those strings appears somehow superposed with the Schlieren texture. In other words, the droplets of ferronematic phase below $\mathrm{T}_{(\mathrm{N}-1)}$ are expelled towards the defect lines of the predominant LC (which exhibit typical colors of a nematic LC under crossed polarizers). We notice also that the diameter of the droplets is controlled by the cooling rate of the quench. Qualitatively, the faster is the cooling, the smaller are the droplets (on average). By careful examination of the droplets under crossed polarizers (figure 1f), we become aware that nematic disinclination lines exist also inside the droplets (black crosses), meaning that there are not made of isotropic phase. When the sample is heated again, the sizes of the droplets start to increase (figure 1g). Then we see the front of isotropic phase (black under crossed polarizers) advancing against the nematic region (figure $1 \mathrm{~h}$ ), until it invades the whole space (figure 1i). Furthermore, it is important to point out that no matter how many temperature cycles are applied to the samples, the magnetic nanoparticles never form a solid flocculate, which means that some LC acts as a solvent inside the droplets.

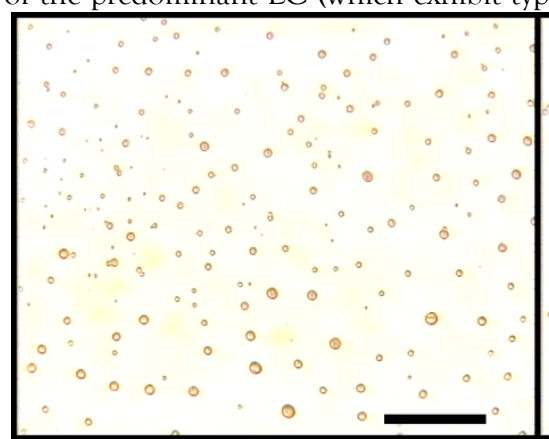

(a)

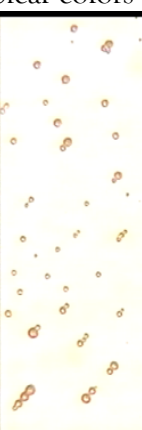

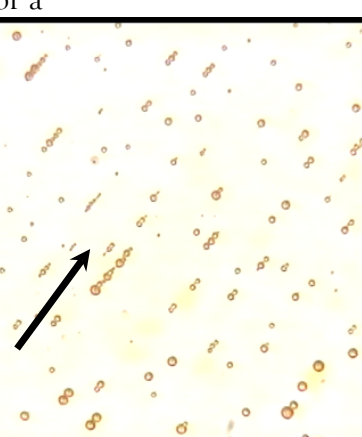

(b)

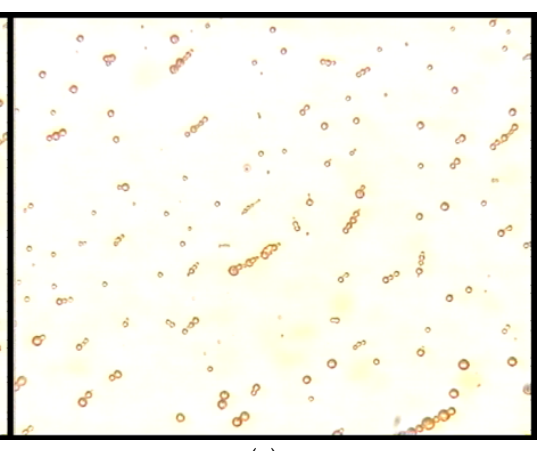

(c)

Figure 2: Application of a magnetic field ( $\mathrm{B}=0.03$ Tesla) to the droplets after the demixing. Polarizers are parallel. The scale bar is 50 $\mu \mathrm{m}$. (a): Before application of the field, spherical droplets are present, with diameters ranging from 1 to $7 \mu \mathrm{m}$ (with a maximum of the distribution around $4 \mu \mathrm{m}$ ); (b): after 9 min of field application, most droplets are found within chains formed by magnetic dipolar attractions; (c): after switching off the field, the droplets remain attached but the orientation of the chains have started to randomize.

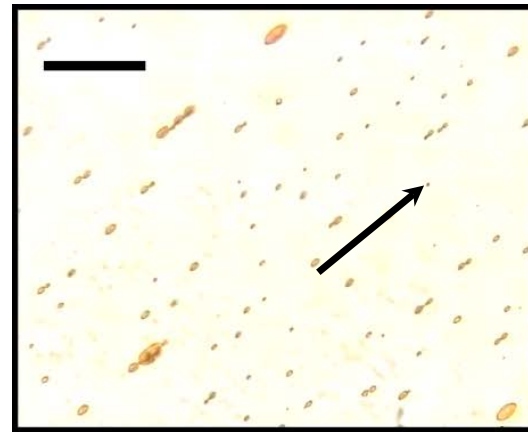

(a)

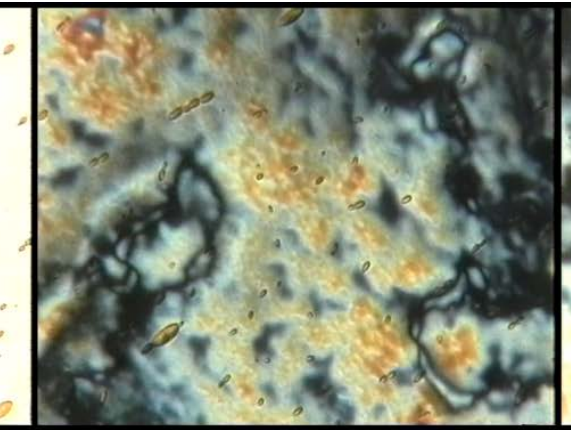

(b)

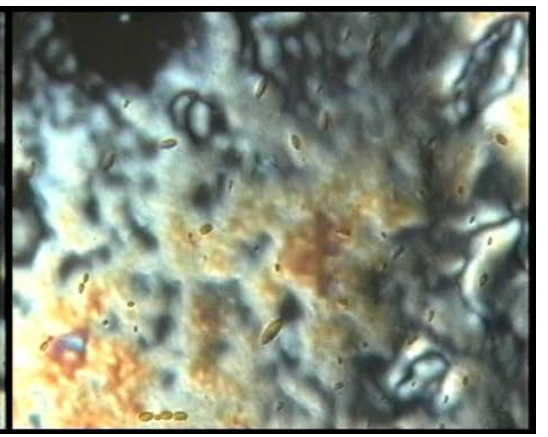

(c)

Figure 3: Application of a magnetic field ( $\mathrm{B}=0.03$ Tesla) since the beginning of the demixing below $\mathrm{T}(\mathrm{N}-\mathrm{I})$ observed under parallel (a) and crossed polarizers $(\mathrm{b}, \mathrm{c})$. (a): the droplets are not only forming chains, but they are also elongated as prolate ellispoids; (b): under crossed polarizers, the orientation of the chains appears independent of the Schlieren texture of the nematic environment; (c): after release of the magnetic field, both chaining and ellipsoidal deformation persist, but they exhibit random orientations.

Following the variance rule, the existence of a bi-phasic state below $\mathrm{T}_{(\mathrm{N}-\mathrm{I})}$ is a direct consequence of a multi-component system. Such a case was reported for hydrophilic silica particles $\left(5 \mathrm{~nm}\right.$ diameter) in a $5 \mathrm{CB}$ host, ${ }^{24}$ that do not disperse in the nematic phase but are captured by the N-I interface at the transition. In that example, a complete exclusion of the silica particles is observed below the I-N transition, leading to a precipitate forming a "cellular" structure. Such a network formation below $\mathrm{T}_{(\mathrm{N}-\mathrm{l})}$ has also been reported recently for the same 5CB LC loaded with sterically stabilized colloids made of a polymer (PMMA), of different diameters ranging from 100 $\mathrm{nm}$ to $1 \mu \mathrm{m} .{ }^{25}$ By using NMR spectroscopy, the authors of this article proved that even $10^{\circ} \mathrm{C}$ below $\mathrm{T}_{(\mathrm{N}-\mathrm{I})}, 10 \%$ of the $5 \mathrm{CB}$ molecules were still in the isotropic phase coexisting with the nematic phase, which they explain by a tri-component system (LC / particles / solvent residue). Here on the contrary, the magnetic nanoparticles accumulate inside non isotropic droplets. The formation of the nematic order below $\mathrm{T}_{(\mathrm{N}-\mathrm{l})}$ tends here to confine the nanoparticles into concentrate ferronematic droplets in coexistence with the apparently unmodified and empty nematic phase.

Microscopic observations of the 2-phase behavior are also made under an applied magnetic field. At first we cool from isotropic to nematic phase at $2{ }^{\circ} \mathrm{C} / \mathrm{min}$ without any field: small spherical droplets form as seen on figure 2a. Under the application of a magnetic field of low intensity (0.03 Tesla) 
This document is the Accepted Manuscript version of a Published Work that appeared in final form in see J. Phys. Chem. B 2005, 109, 14292-14299, copyright (C) American Chemical Society after peer review and technical editing by the publisher. To access the final edited and published work see http://pubs.acs.org/doi/abs/10.1021/jp0455024

parallel to the plane of the sample, the droplets which are close enough to attract each other gather into chains containing between two and fifteen droplets (figure $2 \mathrm{~b}$ ). When the field is released, chains persist for a while but display rotational Brownian motion as can bee seen on figure 2c. Chains also appear flexible as droplets within a given chain can move around and eventually escape from the chain. If we wait longer, all the chains disappear. When heating to isotropic, all droplets disappear and nanoparticles disperse themselves again homogeneously in the isotropic LC.

Another kind of experiment consists in applying the same magnetic field all along the transition from the isotropic to the nematic strate. In that case, the droplets not only chain but although significantly elongate (figure 3a), meaning that they are both magnetic and deformable. However, we never observe droplets coalescing under magnetic dipolar attraction. Observed under crossed polarizers (figure $3 b$ ), the nematic host phase still exhibits disinclination lines, which is a clue that it is poor in magnetic nanoparticles and does not benefit from their orienting properties. Contrary to the strings of droplets under zero field connected to the Schlieren texture, this chaining under a magnetic field is ascribable to fieldinduced dipolar attractions between the highly magnetic droplets. After field release, the chains persist and the droplets keep their elongated shape (figure 3c), but they starts to rotate randomly under Brownian motion.

The interpretation of these results is delicate and needs some discussion compared to previously published works on mixtures of nematics with either solid or liquid colloids. With a somehow analogous system consisting of droplets of an aqueous ferrofluid mixed with an immiscible nematic LC, ${ }^{26}$ Poulin et al. have observed the spontaneous chaining of the droplets by the interplay of surface and elastic forces, in the absence of magnetic field. This effect is not dictated by the magnetic interactions, and therefore is observed also for pure water droplets as long as an appropriate surfactant is used to determine a normal boundary condition of the LC molecules at the surface of the droplets. ${ }^{27}$ When the anchoring energy dominates over the elastic energy (associated to distorsions of the nematic director), each droplet associated to a so-called "hedgehog" defect interacts with the other droplets by (i) long range (dipolar type) attraction and (ii) short range repulsion (of elastic nature, presumably due to the thin nematic layer squeezed when droplets become closer). The situation in the present work is different because $5 \mathrm{CB}$ constitutes both the nematic host matrix and the solvent of the dispersed ferronematic phase. Therefore we can imagine that the boundary conditions at the surface of the droplets is rather planar than normal. At least we know that the interfacial cost of the droplets in the nematic matrix must be low, because they are easily deformed into ellipsoids by a weak magnetic field. This point is corroborated by the absence of coalescence, which would be favored by a larger interfacial tension.

To summarize those optical microscopy experiments, we observe: i) a biphasic behavior below $\mathrm{T}_{(\mathrm{N}-\mathrm{I})}$ with a nematic LC phase poor or empty of nanoparticles and droplets of ferronematic phase rich in nanoparticles; ii) a sensitivity to a weak magnetic field causing the deformation and/or the chaining of the droplets along the field direction, but no coalescence.

DSC experiments
Mixtures with different volume fractions of nanoparticles in 5CB are studied by Differential Scanning Calorimetry in order to determine if the biphasic behavior observed optically leads to an endothermic peak, which is a thermodynamic signature of the nematic to isotropic transition. The curves of the heat flows vs. temperature are collected with a Perkin-Elmer DSC 7 calorimeter coupled with a cryostat at different rates of temperature change.

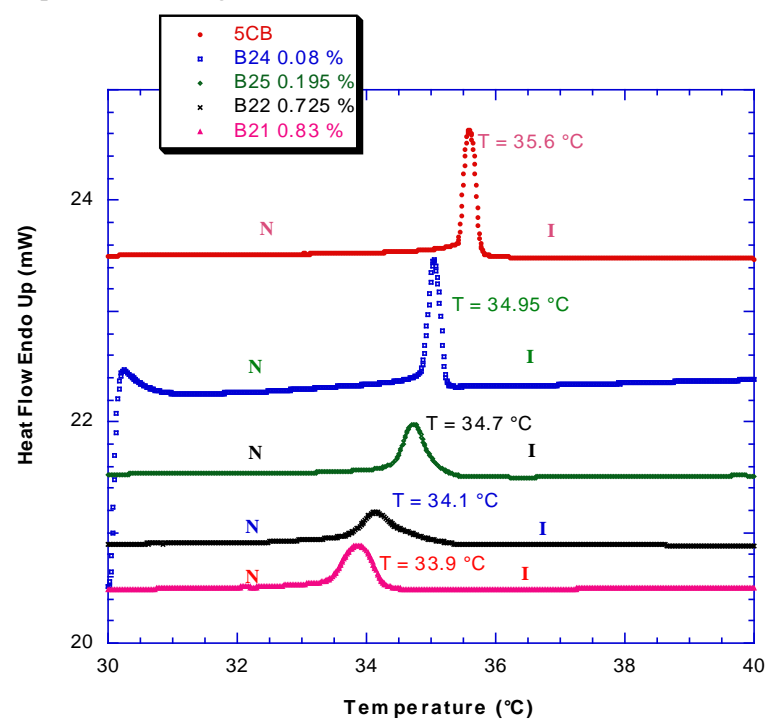

Figure 4: DSC thermograms on heating at $1^{\circ} \mathrm{C} / \mathrm{min}$ for $5 \mathrm{CB}$ ferronematic with different volume fractions of maghemite nanoparticles.

We report here only the results on heating at $1{ }^{\circ} \mathrm{C} / \mathrm{min}$, which is also the rate also used in the SAXS experiments. From these thermograms (figure 4), we see that increasing the quantity of nanoparticles incorporated in the liquid crystal matrix causes a decrease of the range of the nematic phase existence together with a widening of the clarification peak. From this series of peaks, $\mathrm{T}_{(\mathrm{N}-\mathrm{l})}$ measured precisely by DSC is plotted vs. the volume fraction $\Phi$ of nanoparticles on figure 5 .

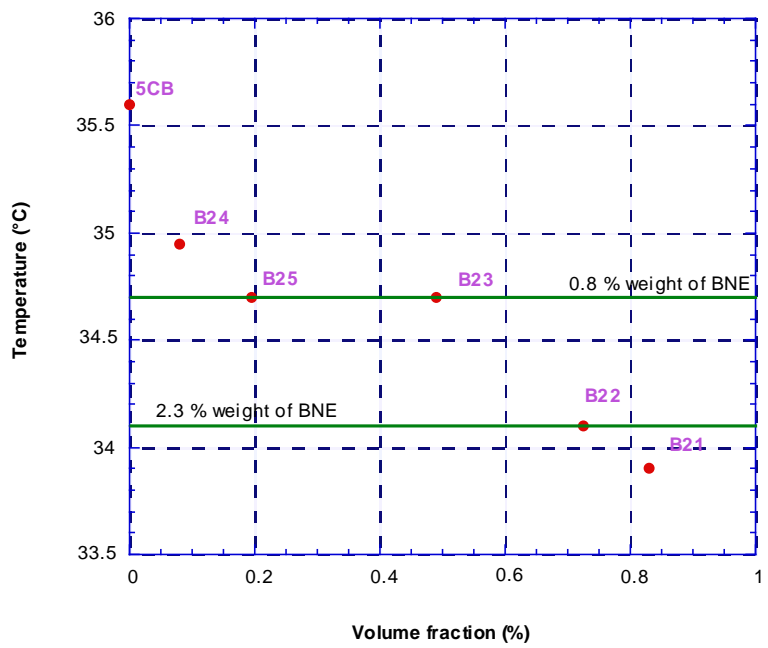

Figure 5: Dependence of the phase transition temperature $\mathrm{T}(\mathrm{N}-\mathrm{I})$ from the nematic phase to the isotropic phase (determined on heating at $1{ }^{\circ} \mathrm{C} / \mathrm{min}$ ) with the volume fraction on nanoparticles. The horizontal lines represent the two control experiments made without nanoparticles but with the indicated concentration of BNE surfactant.

We see that the decrease of $\mathrm{T}_{(\mathrm{N}-\mathrm{I})}$ starts from the lowest value of $\Phi$, which proves that no threshold concentration of particles is required for the 2-phase behavior. From the almost 

access the final edited and published work see http://pubs.acs.org/doi/abs/10.1021/jp0455024

linear decrease of $\mathrm{T}_{(\mathbb{N}-1)}$ as a function of $\Phi$, we conclude that some chemical species is more soluble in the isotropic phase than in the nematic phase and perturbs the nematic phase enough to be considered as an impurity. Two possibilities can be considered: on the one hand, a small amount of nanoparticles can be directly soluble inside the nematic matrix (in addition to the droplets which contain most of the nanoparticles); on the other hand, the nanoparticles could be perfectly insoluble in the nematic phase and only the surfactants desorbed from the nanoparticles enter inside the nematic phase. To decide which phenomenon really occurs, we analyze the thermogram of $5 \mathrm{CB}$ without nanoparticles but with some added BNE surfactant inside. The transition to isoptropic phase occurs at $34.1^{\circ} \mathrm{C}$ and $34.7^{\circ} \mathrm{C}$ respectively with $2.3 \%$ and $0.8 \%$ in weight of BNE, which are the same temperatures as B22 and B25 samples respectively. These values fit really well with the estimates of the final proportion of free BNE inside the ferronematics by dilution of the initial ferrofluid, which contains about 5 wt $\%$ BNE for a volume fraction of nanoparticles $\Phi=1.2 \%$. To be entirely sure that the decrease of $\mathrm{T}_{(\mathrm{N}-\mathrm{l})}$ is due the presence of BNE only, we separate the nematic phase from the magnetic isotropic phase by decantation of the droplets on a strong magnet ( $\sim 0.5$ Tesla). The DSC thermogram of the entire ferronematic and of this separated $\mathrm{N}$ phase showing exactly the same $\mathrm{T}_{(\mathrm{N}-\mathrm{I})}$ temperature lower than for pure $5 \mathrm{CB}$, we are allowed to conclude that the free BNE is the only parameter that regulates this decrease of transition temperature. As a conclusion, we have proved that the magnetic nanoparticles do not contribute to this decrease of $\mathrm{T}_{(\mathrm{N}-\mathrm{l})}$, for which only the free surfactant is responsible. This fact is corroborated by the absence of iron titrated by AAS inside the nematic phase isolated from the droplets by magnetic separation of a ferronematic. If any nanoparticles existed in this nematic phase, the corresponding [Fe] would be less than $10^{-5} \mathrm{molL}^{-1}$ (sensitivity of AAS). Let us note that previous studies on ferronematics with other magnetic nanoparticles coated with surfactants at lower concentrations ( $<0.1$ vol \%) reported a monophasic behavior with no variation of the clarification temperature.

\section{SAXS experiments}

Optical microscopy has indicated that the I-N transition is concomitant with the apparition of a dispersed phase dense in magnetic nanoparticles in coexistence with the nematic host phase containing very few particles or even not at all. Such phase separation can be studied using small angle neutron or X-ray scattering, two techniques that are well suited to study the structure and interactions between nanoparticles in ferrofluids and materials derived from them. SAXS is appropriate here because the contrast between the nematic matrix and the ferric oxide is quite important, so that the signal of $5 \mathrm{CB}$ can be considered as a background scattering (supporting information). The cross-correlations between nanoparticles in ferronematics are pointed out by comparing the intensity scattered by the composite systems to the one of the dilute ferrofluid, behaving as an ideal gas. SAXS experiments are performed on two samples at two different volume fractions $\Phi$ (equal to about 0.2 and $0.7 \%$ respectively) and at different temperatures to follow the interactions between nanoparticles as a function of temperature. These experiments were done in the LURE laboratory using synchrotron radiation (beamline D24, Orsay, France). The samples are introduced in Lindemann X-ray glass capillaries of
$1 \mathrm{~mm}$ inner diameter placed horizontally in a Mettler F82 temperature stage. Different scattering patterns in transmission mode are recorded as a function of temperature with increasing or decreasing rates of $\pm 1{ }^{\circ} \mathrm{C} / \mathrm{min}$. The distance from the sample to the detector was $1750 \mathrm{~mm}$ enabling to cover the following q-range : $9 \cdot 10^{-3}-2 \cdot 10^{-1} \AA^{-1}$. Each SAXS curve is the average of four independent frames, each obtained with an acquisition time of $100 \mathrm{~s}$.

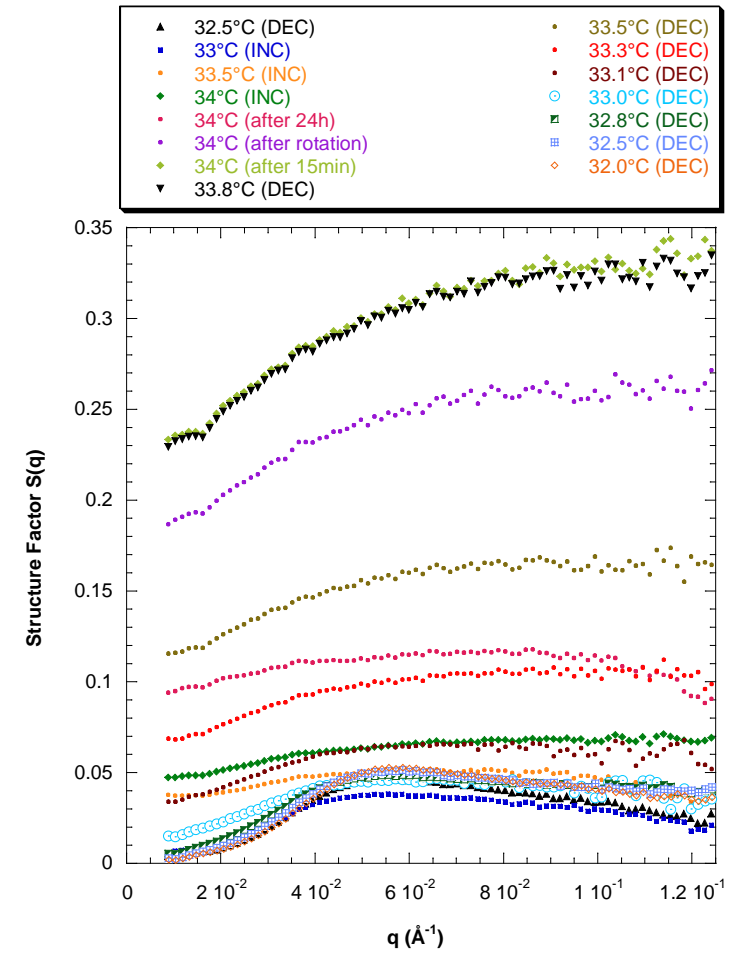

Figure 6: Curves showing the dependence of the structure factor of nanoparticles at different temperatures with a rate of $1{ }^{\circ} \mathrm{C} / \mathrm{min}$ as a function of increasing (INC) or decreasing (DEC) temperature for the more concentrated sample (volume fraction $=0.725 \%)$. ${ }^{*}$ capillary turned around its symmetry axis N. B.: We notice from the graphs that the intensity is higher when the sample is in the isotropic state and decreases regularly when reaching the isotropic-nematic transition. The opposite phenomenon is observed in the reverse way. We think that it is due to the sedimentation of the droplets to the bottom of the capillary, because they are rich in nanoparticles and thus with a higher mass density than the nematic phase below $\mathrm{T}_{\mathrm{C}}$. That would result in a lower concentration of nanoparticles analyzed in the beam, and so a lower scattered intensity. As control experiments, we let first the sample at rest for $24 \mathrm{~h}$ and we observe the intensity slowly increasing by spontaneous homogenization; then, we rotate the capillary by $180^{\circ}$ around its axis, and we check that the intensity further increases by a factor three (see curves at $34^{\circ} \mathrm{C}$ ). This variation of nanoparticles concentration in the beam due to sedimentation of bi-phasic samples is also responsible for the difference between the plateau value $\mathrm{S}(\mathrm{q})$ at large $\mathrm{q}$ and the expected value of 1 .

First of all we determine the form factor $I_{\mathrm{FF}}(\mathrm{q})$ in a stable dilute ferrofluid, which represents intra-particle density correlations due to their shape convolved with the polydispersity of sizes. The background signal $I_{\mathrm{Bk}}(q)$ without capillary is also recorded. To take in consideration the contribution $I_{\mathrm{LC}}(q)$ of the liquid crystal to the signal $I_{\mathrm{FN}}(q)$ of the ferronematics, we record the intensity scattered by pure $5 \mathrm{CB}$ at different temperatures between $33^{\circ} \mathrm{C}$ and $36^{\circ} \mathrm{C}$. The 
This document is the Accepted Manuscript version of a Published Work that appeared in final form in see J. Phys. Chem. B 2005, 109, 14292-14299, copyright (C) American Chemical Society after peer review and technical editing by the publisher. To access the final edited and published work see http://pubs.acs.org/doi/abs/10.1021/jp0455024

signals obtained at all temperatures are strictly identical and thus can be averaged together. In the studied q-range $I_{\mathrm{LC}}(q)$ appears independent of temperature on each side of N-I transition because the signature of the nematic order occurs at higher $q$ values. In our experiments, the liquid crystal only contributes to the high-q background and thus can be subtracted (supporting information) to extract the signal of nanoparticles only. All transmitted X-ray intensities $T_{\mathrm{Bk}}, T_{\mathrm{LC}}$, $T_{\mathrm{FF}}$ and $T_{\mathrm{FN}}$, are measured with an air ionization chamber. As a result, the structure factor is calculated at all temperatures using the following equation:

$$
\mathrm{S}(\mathrm{q})=\frac{\frac{\mathrm{I}_{\mathrm{FN}}(\mathrm{q})}{\mathrm{T}_{\mathrm{FN}} \Phi_{\mathrm{FN}}}-\frac{\mathrm{I}_{\mathrm{LC}}(\mathrm{q})}{\mathrm{T}_{\mathrm{LC}}}}{\frac{\mathrm{I}_{\mathrm{FF}}(\mathrm{q})}{\mathrm{T}_{\mathrm{FF}} \Phi_{\mathrm{FF}}}-\frac{\mathrm{I}_{\mathrm{Bk}}(\mathrm{q})}{\mathrm{T}_{\mathrm{Bk}}}} \text { where } \Phi_{\mathrm{FF}} \text { and } \Phi_{\mathrm{FN}} \text { are the }
$$

volume fractions of nanoparticles in ferrofluid and ferronematic respectively, measured as previously explainedErreur! Signet non défini.

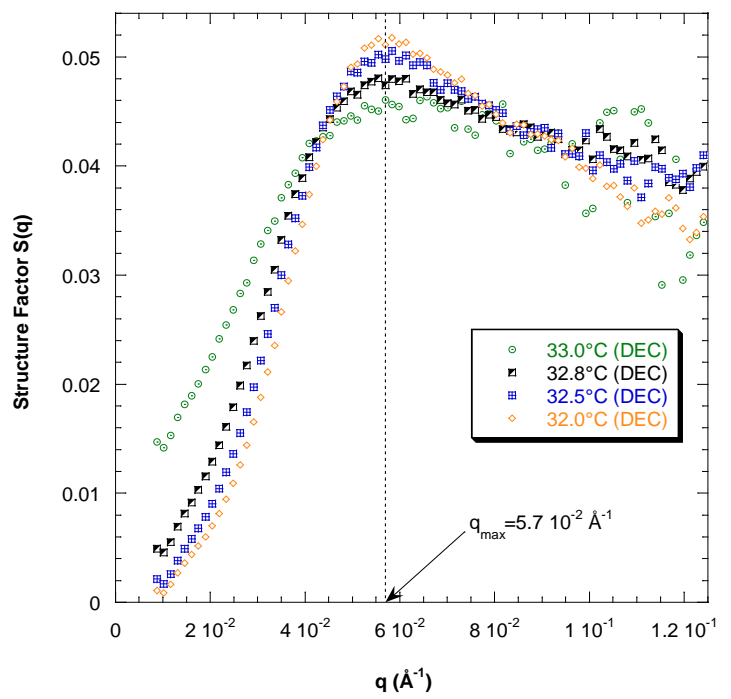

Figure 7: Zoom close to the Isotropic-Nematic transition for the more concentrated sample (volume fraction $=0.725 \%$ ).

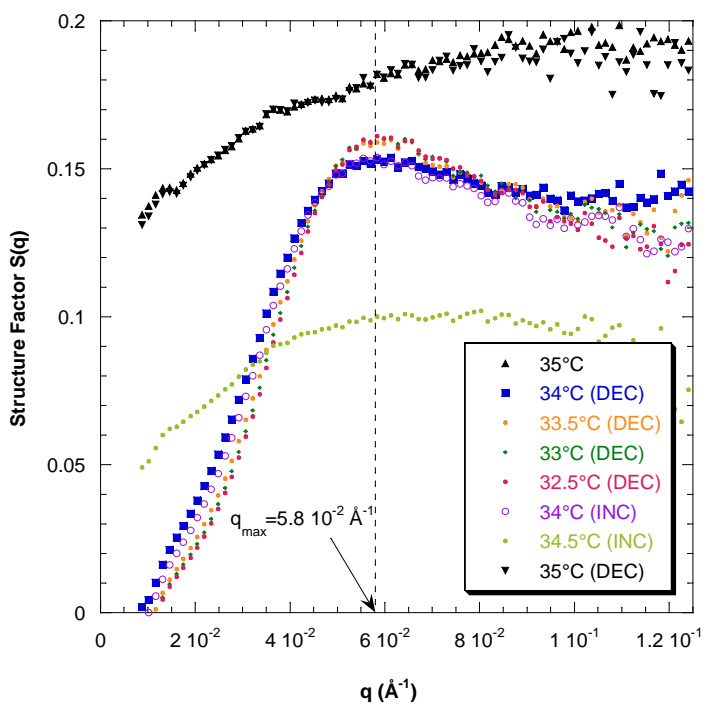

Figure 8 : Curves showing the dependence of the structure factor of nanoparticles at different temperatures with a rate of $1{ }^{\circ} \mathrm{C} / \mathrm{min}$ on increasing (INC) or decreasing (DEC) temperature for the less concentrated sample (volume fraction $=0.195 \%$ ).
By this way, the structure factor of the nanoparticles is determined as a function of temperature for two samples with a volume fraction of nanoparticles of $0.725 \%$ and $0.195 \%$ respectively. These SAXS experiments being sensitive mainly to the nanoparticles, they provide a signature of the $\mathrm{I}$ to $\mathrm{N}$ transition specific to the nanoparticles, pointing out the effect of the transition of the LC matrix on their spatial organization.

At high temperature where the sample is in the isotropic and homogeneous state, S(q) is nearly flat except at lowest q values where it slightly decreases, which tells us about repulsive interactions between nanoparticles (low compressibility). When temperature is decreased below a threshold $T_{C}$, a correlation peak appears suddenly. From the top of this peak at the abscissa $q_{\max }$, we deduce the more likely distance between the particles $d_{\max }=2 \pi / q_{\max }$. Thus we obtain an estimate of the local volume fraction $\Phi_{\text {local }}=\frac{\pi \frac{\mathrm{d}_{0}^{3}}{6}}{\mathrm{~d}_{\max }^{3}}, d_{0}$ being the characteristic diameter of the nanoparticles that we estimate by $d_{0}^{\text {SAXS }}$ obtained from the form factor (see Supporting information).

For the more concentrated sample $(0.7 \%)$ a local volume fraction of $17 \%$ is obtained and for the less concentrated one $(0.2 \%), 18 \%$. The value of $\Phi_{\text {local }}$ does not vary significantly with the average $\Phi$ measured by iron titration and is much higher than $\Phi$. Therefore we deduce that $\Phi_{\text {local }}$ measured by SAXS is the volume fraction of nanoparticles inside the droplets observed by optical microscopy below $\mathrm{T}_{(\mathrm{N}-\mathrm{I})}$. As a consequence, the $T_{C}$ temperature defined as the appearance of the correlation peak is ascribable to the appearance of the droplets and therefore directly related to $\mathrm{T}_{(\mathrm{N}-\mathrm{l})}$ of the ferronematic.

For a given sample, reproducible results for $\mathrm{T}_{\mathrm{C}}$ were obtained when increasing and decreasing temperature several times, independently of the sample history. Besides, the two samples do not indicate the same temperature $T_{C}$ : for the more concentrated sample $\mathrm{T}_{\mathrm{C}}=32.8-33^{\circ} \mathrm{C}$ (figure 7), whereas for the less concentrated sample, $\mathrm{T}_{\mathrm{C}}=34-34.5^{\circ} \mathrm{C}$ (the range of transition is not broader but we recorded less spectra around the transition, figure 8 ). This dependence of $T_{C}$ with the average concentration of particles is in agreement with the variation of the clarification temperature obtained by DSC thermograms : $\mathrm{T}_{(\mathrm{N}-1)}=34.1^{\circ} \mathrm{C}$ at $\Phi=0.7 \%$ and $\mathrm{T}_{(\mathrm{N}-\mathrm{l})}=34.7^{\circ} \mathrm{C}$ at $\Phi=0.2 \%$ (figure 5). Even though the slight differences (0.4-1 ${ }^{\circ} \mathrm{C}$ ) between $\mathrm{T}_{\mathrm{C}}$ and $\mathrm{T}_{(\mathrm{N}-\mathrm{I})}$ are likely ascribable to an experimental difference between the temperature stages used for each method, we cannot conclude for sure to the equivalence of $\mathrm{T}_{\mathrm{C}}$ and $\mathrm{T}_{(\mathrm{N}-\mathrm{I})}$. Nevertheless, the results of these two techniques converge to the same conclusion that both temperatures $T_{C}$ and $T_{(\mathbb{N}-1)}$ decrease simultaneously. These temperature shifts are related to the concentration of free BNE soluble in the nematic phase.

\section{CONCLUSION}

Colloidal dispersions of small particles in nematic liquid crystals are an interesting type of soft matter. The difference from ordinary colloids arises from the orientational ordering of the calamitic molecules and the resulting structure in the colloid. In this work, we present a detailed study of the phase behavior of $5 \mathrm{CB}$ with incorporated magnetic nanoparticles by different techniques including optical microscopy, DSC, and SAXS. We have found a multi-scale organized system: magnetic nanoparticles of maghemite confined inside ferronematic droplets, themselves dispersed in a nematic host. By 
This document is the Accepted Manuscript version of a Published Work that appeared in final form in see J. Phys. Chem. B 2005, 109, 14292-14299, copyright (C) American Chemical Society after peer review and technical editing by the publisher. To access the final edited and published work see http://pubs.acs.org/doi/abs/10.1021/jp0455024

introducing a large amount of magnetic nanoparticles in a nematic host, we have found indeed a phase separation between droplets rich in nanoparticles and a continuous nematic LC empty of nanoparticles and containing unadsorbed surfactant. This 2-phase behavior has never been reported in previous works, certainly because authors used much lower particles concentrations as ours ( $\Phi$ of $5 \times 10^{4} \%$ at most against $\Phi>0.2 \%$ here). We have demonstrated that the transition temperatures $\mathrm{T}_{(\mathrm{N} \cdot \mathrm{l})}$ measured by DSC and $\mathrm{T}_{\mathrm{C}}$ by SAXS are both affected by the presence of nanoparticles and surfactant concentration in a similar way, which is a decrease. The DSC measurements show a decrease of the $T_{(\mathrm{N}-1)}$ temperature linear with $\Phi$ as well as a widening of the corresponding peak. These two elements lead us to conclude on the partial solubilization of some species in the liquid crystal in the nematic phase, which we have identified to be pure surfactant. Concerning the possible discrepancy between $\mathrm{T}_{\mathrm{C}}$ associated to the presence of droplets and $\mathrm{T}_{(\mathrm{N}-1)}$ related to the nematic host phase, experiments could be attempted in future on an experimental set-up combining SAXS and DSC simultaneously. Other perspectives to be explored could be: using the dipolar forces between the magnetic droplets under field to probe the elasticity of the nematic matrix; checking the existence of a hysteresis loop in the magnetization curve of ferronematics as predicted theoretically, ${ }^{1}$ obtaining true homogeneous ferronematics by controlling the anchoring of the LC on the nanoparticles. This last goal could be reached by grafting mesogenic molecules resembling to the matrix. More generally, the role of the interaction between the "surfactant" molecules covering the nanoparticles and the thermotropic molecules of the matrix is of prime importance and will be clarified in a forthcoming study; this needs to coat the magnetic nanoparticles by several types of molecules that will interact differently with $5 \mathrm{CB}$.

\section{Supporting information:}

The distribution of the diameters of the nanoparticles used in this study is determined by fitting either the magnetization curve or the form factor of the dilute ferrofluid. As ferrofluids are giant paramagnetic liquids indeed, their magnetization curve versus the applied magnetic field follows a Langevin's law, ${ }^{28,29}$ as long as their interactions are negligible i. e. for small volume fractions $\Phi<$ $1 \%$. The measured magnetization is highly sensitive to the distribution of diameters of the magnetic nanoparticles in the sample, ${ }^{30,31}$ for which we assume a Log-normal distribution law calculated from: $\mathrm{P}(\mathrm{d})=\frac{1}{\sqrt{2 \pi} \sigma d} \exp \left[-\frac{1}{2 \sigma^{2}}\left(\ln \frac{d}{d_{0}}\right)^{2}\right]$

with a characteristic diameter $\mathrm{d}_{0}=\exp \langle\ln \mathrm{d}\rangle$ and a standard deviation $\sigma$. Combining the Langevin's law with the distribution $\mathrm{P}(\mathrm{d})$ provides us with two parameters $\mathrm{d}_{0}$ and $\sigma$ used to fit the experimental curves. The same Log-normal distribution law is also used to compute the theoretical form factor of the dilute ferrofluid, assumed to be a polydisperse assembly of spherical nanoparticles. By adjusting the magnetization curve and the experimental form factor of the ferrofluid used in this study, the characteristic diameters $d_{0}{ }^{\mathrm{mag}}$ $=6.3 \mathrm{~nm}$ and $d_{0}^{\text {SAXS }}=7.6 \mathrm{~nm}$ are found respectively, both with $\sigma=0.4$ corresponding to a relatively broad distribution. The discrepancy between the two methods originates from the magnetically disordered shell at the surface of the grains, which contributes to X-rays scattering but not to magnetization. In other words, the magnetic core of the nanoparticles is somehow smaller than their geometrical volume.

a)

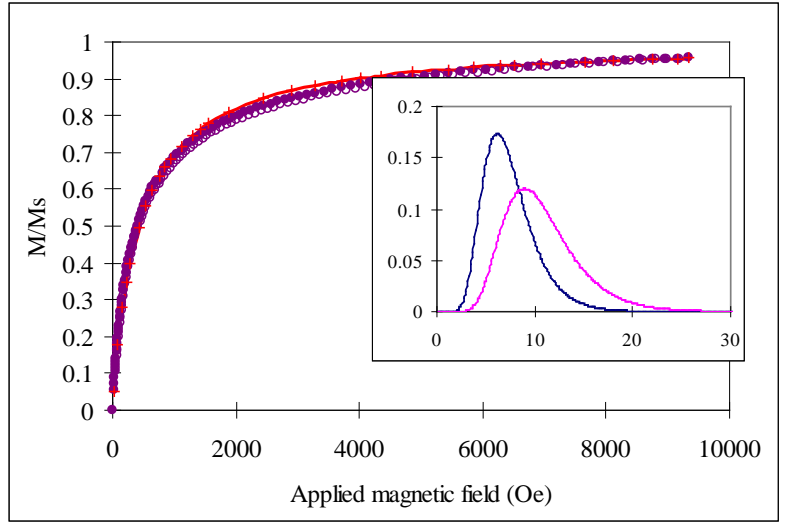

b)

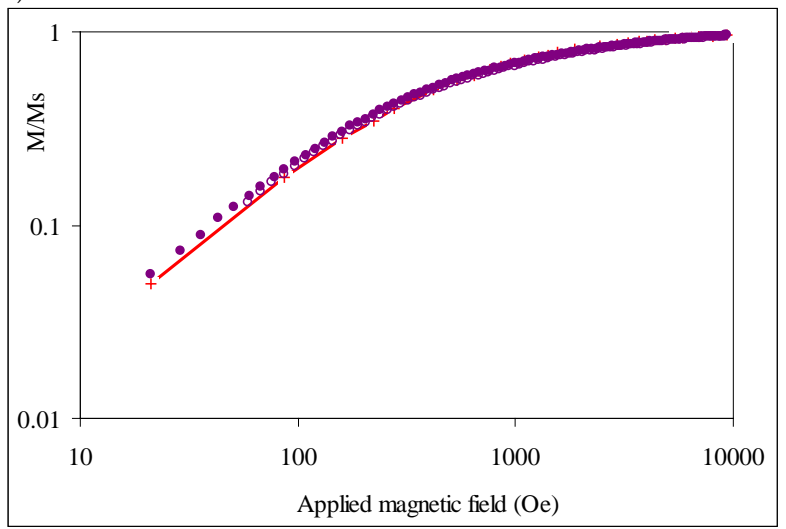

Supporting information 1: Magnetization curve of the ferrofluid in dichloromethane measured with a homemade vibrating sample magnetometer. The magnetization $M$ is normalized by the value at saturation $M_{s}=42$ Oe is plotted vs. the applied magnetic field $H$ (Oe). The plateau value corresponds to a volume fraction $\Phi=\mathrm{M}_{\mathrm{s}} / \mathrm{m}_{\mathrm{s}}=1.2 \%$ where $m_{\mathrm{s}}=3400$ Oe is the specific magnetization of colloidal maghemite. ${ }^{32}$ The two parameters $\mathrm{d}_{0}$ and $\sigma$ describing the size polydispersity enable to fit the magnetization at both high (a) and low (b) fields.

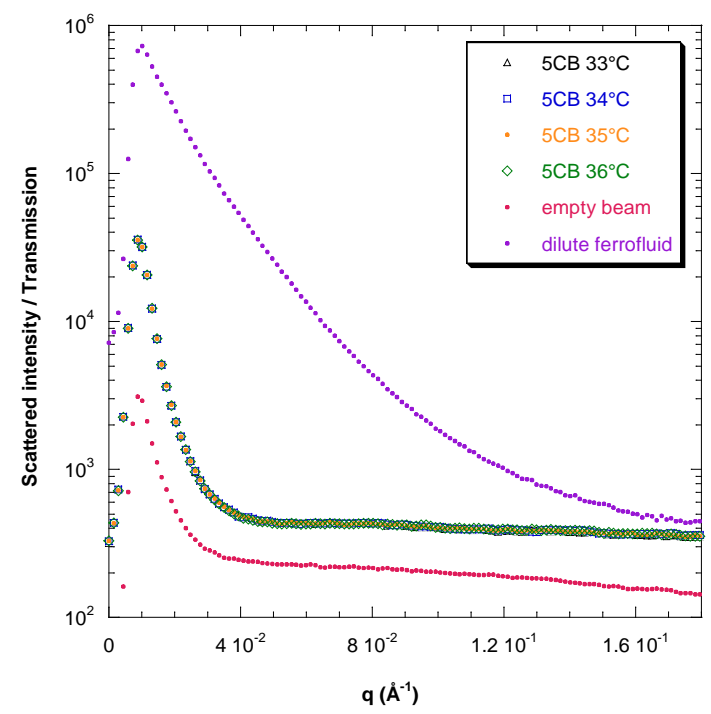

Supporting information 2: SAXS intensities corrected by sample transmission as a function of the scattering vector $\mathrm{q}$ for 
This document is the Accepted Manuscript version of a Published Work that appeared in final form in see J. Phys. Chem. B 2005, 109, 14292-14299, copyright (C) American Chemical Society after peer review and technical editing by the publisher. To access the final edited and published work see http://pubs.acs.org/doi/abs/10.1021/jp0455024

the empty beam, the dilute ferrofluid and the liquid crystal $5 \mathrm{CB}$ alone at different temperatures around $\mathrm{T}_{(\mathrm{N}-1)}$.

\section{Acknowledgements:}

We wish to thank C. Bourgaux as our local contact in the LURE synchrotron facility and D. Talbot who performed the AAS measurements. C. Da Cruz also thanks J. Malthête for the access to DSC technique at the Curie Institute.

\section{References}

${ }^{1}$ F. Brochard, P. G. de Gennes, J. Phys. (Paris), 1970, 31, 691.

2 J. C. Loudet, P. Barois, P. Poulin, Nature, 2000, 407, 611.

${ }^{3}$ H. Stark, Eur. Phys. J. B, 1999, 10, 311.

${ }^{4}$ T. C. Lubensky, D. Pettey, H. Currier, H. Stark, Phys. Rev. E, 1998, 57, 610.

${ }^{5}$ B. I. Lev, P. M. Tomchik, Phys. Rev. E, 1999, 59, 591.

${ }^{6}$ P. Poulin, H. Stark, T. C. Lubensky, and D. A. Weitz, Science, 1997, 275, 1770.

${ }^{7}$ P. Poulin, D. A. Weitz, Phys. Rev. E, 1998, 57, 626.

${ }^{8}$ V. J. Anderson, E. M. Terentjev, S. P. Meeker, J. Crain, W. C. K. Poon, Eur. Phys. J., 2001, 4, 11.

${ }^{9}$ V. J. Anderson, E. M. Terentjev, Eur. Phys. J., 2001, 4, 21.

${ }^{10}$ S. P. Meeker, W. C. K. Poon. J. Crain, and E. M. Terentjev, Phys. Rev. E, 2000, 61, R6083.

11 P. G. de Gennes, The Physics of Liquid Crystals, 1974, Clarendon, Oxford.

${ }^{12}$ V. Berejnov, V. Cabuil, R. Perzynski, Y. Raikher, J. Phys. Chem. B, 1998, 102, 7132-7138.

${ }^{13}$ J. C. Bacri, A. M. Figueiredo Neto, Phys. Rev. E, 1994, 50, 3860-3864

${ }^{14}$ M. Magalhaes, A. M. Figueiredo Neto, A. Bee, A. Bourdon, J. Chem. Phys., 2000, 113, 10246-10251.

${ }^{15}$ D. Walton, S. M. Shibli, J. Mag. Mag. Mat., 2001, 226/230, 1948-1950.

${ }^{16}$ S. H. Chen, N. M. Amer, Phys. Rev. Lett., 1983, 51, 2298 2301.

17 a) M. Koneracka, V. Kellenerova, P. Kopcansky, T. Kuczynski, J. Mag. Mag. Mat., 1995, 140/144, 1455-1456. b) M. Koneracka, V. Zavisova, P. Kopcansky, J. Jadzyn, G. Czechowski, B. Zywucki, J. Magn. Magn. Mat., 1996, 157/158, 589-590. c) I. Potocova, M. Koneracka, P. Kopcansky, M. Timko, L. Tomco, J. Jadzyn, G. Czechowski, J. Magn. Magn. Mat., 1999, 201, 163-166. d) I. Potocova, M. Koneracka, P. Kopcansky, M. Timko, L. Tomco, J. Jadzyn, G. Czechowski, J. Magn. Magn. Mat., 1999, $196 / 197,578-580$

${ }^{18}$ R. E. Rosensweig, US Patent no 3917 538, 1975.

${ }^{19}$ R. Massart, C. R. Acad. Sci. , Ser. C, 1980, 291, 1.

${ }^{20}$ R. Massart, IEEE Trans. Magn., 1981, MAG 17, 1247-1248.

${ }^{21}$ S. Lefebure, E. Dubois, V. Cabuil, S. Neveu, R. Massart, J. Mater. Res., 1998, 13, 2975-2981.

${ }^{22}$ Beycostat NE (or N B09) is a commercial product provided by CECA, Courbevoie (France) used to disperse inorganic pigments in aromatic or chlorinated oils (but insoluble in aliphatic solvents).

${ }^{23}$ The relationship between molar concentration in iron and volume fraction of nanoparticles is $\Phi(\%)=1.577[\mathrm{Fe}]\left(\mathrm{mol} . \mathrm{L}^{-1}\right)$ taking into account the molecular weight and the mass density of iron oxide.

24 J. L. West, A. Glushchenko, G. Liao, Y. Reznikov, D. Andrienko, M. P. Allen, Phys. Rev. E, 66, 012702, 2002.

${ }^{25}$ D. Vollmer, G. Hinze, B. Ullrich; W. C. K. Poon, M. E. Cates, A. B. Schonfield, Langmuir 2005, 21, 4921-4930.

${ }^{26}$ P. Poulin, V. Cabuil, D. A. Weitz, Phys. Rev. Lett., 1997, 79, 4862-4865.

${ }^{27}$ P. Poulin, D. A. Weitz, Phys. Rev. E, 1998, 57, 626-637.
28 R. E. Rosensweig, Ferrohydrodynamics, 1985, Cambridge University Press, Cambridge.

${ }^{29}$ I. S. Jacobs, C. P. Bean, Magnetism, a treatise on modern theory and materials, 1963, vol. III, ed. G. T. Rado and H. Suhl, Academic Press.

${ }^{30}$ Magnetic Fluids and Applications Handbook, 1996, edited by Berkovski, Begell House, New York.

${ }^{31}$ R. W. Chantrell, J. Popplewell, S. W. Charles, Physica B and C, 1977, 86-88B, 1421.

32 J.-C. Bacri, R. Perzynski, D. Salin, V. Cabuil, R. Massart, J. Magn. Magn. Mat., 1986, 62, 36-46. 